\title{
A Practical Consensus Guideline for the Integrative Treatment of Parkinson's Disease in Shanghai, China
}

\author{
Weidong Pan ${ }^{\mathrm{a}}$ Jun Liu ${ }^{\mathrm{e}}$ Xiangjun Chen ${ }^{\mathrm{h}}$ Qiudong Wang ${ }^{j}$ Yuncheng $\mathrm{Wu}^{\mathrm{f}}$ \\ Yu Bai ${ }^{b}$ Yi Liu $^{c}$ Wentao Lic Wenwei Li Canxing Yuand Weiguo Houk \\ Xiaoying $\mathrm{Bi}^{\mathrm{k}}$ Jianhua Zhuangl Qin Dong ${ }^{g}$ Dingfang Cai ${ }^{i}$ \\ Specialized Committee of Neuroendocrinology and Specialized Committee of \\ Neurology, Shanghai Association of Chinese Integrative Medicine; \\ Subcommittee of Neurology, Shanghai Chinese Medicine Society \\ ${ }^{a}$ Department of Neurology, Shuguang Hospital, ${ }^{b}$ Department of Neurology, Putuo District \\ Center Hospital, ' Department of Neurology, Shanghai Hospital of Traditional Chinese \\ Medicine, and d Department of Neurology, Longhua Hospital, Shanghai University of \\ Traditional Chinese Medicine, ${ }^{e}$ Department of Neurology, Ruijin Hospital, ${ }^{\text {f }}$ Department of \\ Neurology, Shanghai First Hospital, and 9 Department of Neurology, Renji Hospital, \\ Shanghai Jiaotong University, ${ }^{\text {h}}$ Department of Neurology, Huashan Hospital, and \\ 'Laboratory for Neurology, Institute of Integrative Medicine, Zhongshan Hospital, Fudan \\ University, jDepartment of Integrative Neurology, Pudong Hospital of Traditional Chinese \\ Medicine, and ${ }^{\mathrm{k}}$ Department of Neurology, Changhai Hospital, and 'Department of \\ Neurology, Changzheng Hospital, Third Military Medical University, Shanghai, China
}

\section{Key Words}

Consensus guideline · Parkinson's disease $\cdot$ Integrative medicine $\cdot$ Traditional Chinese medicine - Complementary and alternative medicine - Acupuncture - Tai chi chuan · Yoga ·

Preventive treatment

\begin{abstract}
Integrative medicine, including traditional Chinese medicine (TCM), is a new concept in clinical practice for the treatment of neurodegenerative and most chronic diseases. However, integrative consensus or a guideline for the management of patients with Parkinson's disease (PD) is still lacking. The aim of this paper is to provide a review of experiences in clinical practice from Chinese neurologists and physicians (including TCM doctors) in Shanghai, China, and try to provide a clinical guideline for the treatment of the complex and progressive disease of PD with integrative medicine. We included the following treatments: common Western medication, surgery, TCM decoction and patent medicines, acupuncture and Tui na, Yoga, Tai chi chuan, hyperbaric oxygenation, rehabilitation, and other complementary and alternative
\end{abstract}


Pan et al.: A Practical Consensus Guideline for the Integrative Treatment of Parkinson's Disease in Shanghai, China

medicines for the integrative management of the disease. Neurologists and physicians in Shanghai, China, and all over the world should pay attention to integrative medicine, which may be a good choice of treatment for PD.

(C) 2015 S. Karger AG, Basel

\section{Background}

Integrative treatment for Parkinson's disease (PD) such as dopaminergic therapy, neuroprotective therapy, and traditional Chinese medicine (TCM) might be the unmet medical needs to slow down or stop disease progress $[1,2]$. The current therapies include Western medicine (oral medicine), surgery, physical therapy (kinesiotherapy), psychological counseling, and nursing care, whereas traditional medicines provide beneficial effects on symptoms that help to control the classic motor features of the disease (i.e., tremor, rigidity, and bradykinesia) as well as the non-motor symptoms, including autonomic disturbance, dementia, sleep disturbance, psychiatric disorders, and depression; however, eventually, intolerable disability and invalid clinical effects develop in most patients with PD [3]. Over the past 3 decades, significant progress has been achieved in the diagnosis and treatment of PD in China. Not only TCM but also other integrative medicine proved more and more important in treating the disease in Shanghai, China [4]. Movement disorder clinics and associated centers only exist in part of the tertiary hospitals, and the resources cannot satisfy the need of most patients with PD [5]. In many cases, both general neurologists and physicians are responsible for the diagnosis and treatment of PD patients and are sometimes treating them without adhering to the guidelines. For almost 5 years, the management of PD has been improving. A consensus was prepared according to the guideline from the Chinese Parkinson's Disease and Movement Disorders Society for the management of PD (third edition) [6] which summarized the integrative clinical research in China [4, 7-10]. It is important that neurologists and TCM doctors offer more effective and reasonable integrative treatment to their patients. However, the opinions of physicians, general neurologists, and movement disorder specialists in the clinical practice for the treatment of $P D$ are diverse, which will have a significant influence on the actual benefits of patients suffering from the disease.

\section{Principle of Treatment}

\section{Integrative Treatment}

Most patients with PD have motor and/non-motor symptoms in the early or later stages of the disease. These symptoms not only influence the ability to work but also the activities of daily living (ADL). PD cases should be treated individually, including oral medicine, operations, exercise, psychotherapy, and health care as effective treatment options. Oral medicine is the main treatment choice, and surgery, such as deep brain stimulation (DBS), may be initiated as independent therapy or a complementary and alternative method to assist oral medicine [11, 12]. So far, all therapies have improved the symptoms of PD, but they could not stop disease progression. We should thus focus on the long-term management of PD in order to extend the treatment period, delay disease progression, and decrease the side effects of medical therapy.

\section{Principle of Medication}

Both motor and non-motor symptoms can influence the ADL of patients suffering from PD, and neurologists or physicians should aim to control the symptoms early, thus improving the ability to work and the ADL. Early diagnosis and early medication can improve symptoms, 
delay progression, and prevent motor fluctuation and dyskinesia. The compound levodopa or dopamine receptor agonist used for the treatment of motor symptoms should be started at a small dosage and increased slowly according to the progression of the disease. Individual medication management may be more effective and lessen the side effects with regard to tremor (with/without), dementia (with/without), age at morbidity, occupation, combinative diseases, potential side effects, aspiration, and the economic situation of the patients. Dopaminergic treatment should not be stopped abruptly in order to avoid dopaminergic dysregulation syndrome [6].

\section{Internal Medicine (Oral Medication)}

According to its severity, PD has been divided into two stages - the early stage (HoehnYahr 1-2.5), and the middle and severe stages (Hoehn-Yahr 3-5).

\section{PD Patients in the Early Stage}

As evidence indicates that the development of PD in the early stage is faster than in the middle and severe stages $[13,14]$, it is necessary to treat PD patients with oral medication in the early stage, which includes Western medicine and/or TCM and/or other complementary and alternative medicine (CAM). Dopaminergic medicine and/or monoamine oxidase B inhibitors, vitamin E, herbs, complementary nutrition, and even physical therapy (kinesiotherapy), psychological counseling, and nursing care can be used. The aim of the treatment is to improve the mild movement disorders, such as rigidity, tremor, and some non-motor symptoms, and delay disease progression.

In the case of early-onset PD without dementia, we suggest that neurologists or physicians use a non-egret dopamine receptor agonist, such as pramipexole, piribedil, and ropinirole, monoamine oxidase B inhibitors, such as selegiline and rasagiline, adamantanamine, or compound levodopa, such as Madopar or Sinemet combined with or without entacapone [6]. If the symptom of tremor cannot be controlled, benzhexol is also a good choice. Most tonifying TCM components or patent medicines can be used in treating this stage of PD, such as 'Liu-Wei-Di-Huang-Wan' (六味地黃丸), 'Zhen-Gan-Xi-Feng-Tang' (鎮肝熄風湯), 'TianMa-Gou-Teng-Yin' (天麻勾藤飲), 'Ma-Ren-Zi-Pi-Wan' (麻仁滋脾丸), 'Cong-Rong-Tong-BianKou-Fu-Ye' (蓯蓉通便口服液), and others [4, 12].

In the case of later-onset PD, cognitive impairment, or dementia, the compound levodopa is the first-choice treatment. With progression of the disease, dopamine receptor agonist, monoamine oxidase B inhibition, or compound levodopa combined with entacapone are beneficial choices. Cholinergic resistance should be avoided, especially for elderly, later-onset PD patients with cognitive impairment [6].

\section{PD Patients in the Middle and Severe Stages}

Symptoms will become more complex for patients in the middle and severe stages, due to the development of the disease, the side effects of medication, and motor complications such as motor fluctuation and dyskinesia. The scope of treatment in these stages is to improve the movement disorders, motor complications, and non-motor symptoms.

Motor fluctuations such as end-of-dose deterioration, on-off phenomenon, and dyskinesia (abnormal involuntary movements) are common motor complications in the severe stages of PD. Changing the kind of medication as well as modifying the dose and dosing time may help to improve the complex symptoms. Insect TCM drugs such as stiff silkworm (僵䖯), earthworm (地龍), scorpio (全蝎), and centipede (蜈蚣), as well as tonifying 'Gan' (liver) and 'Shen' (kidney) TCM herbs are helpful in modifying the doses and dosing times of Western medicine adminis- 
Pan et al.: A Practical Consensus Guideline for the Integrative Treatment of Parkinson's Disease in Shanghai, China

tered to these patients. TCM doctors not only use oral TCM decoction or patent medicine, but they also individually use TCM as external application to improve complex complications. Sometimes, DBS, Tai chi chuan, and acupuncture can be used as additional non-drug treatments.

\section{Traditional Chinese Medicine}

\section{The Theory of TCM in PD Treatment}

TCM has gained increasing attention in the treatment of PD. Although it is not curative, it may play a significant role in delaying disease progression and reducing the side effects of Western medicine, as well as improving the patients' ability to perform their ADL $[7,8]$. TCM ameliorates various symptoms, particularly the age-related symptoms that are called 'Shen $\mathrm{xu}^{\prime}$ (kidney deficiency) in Chinese. 'Shen' (the kidney) denotes a functional visceral system ('Zang') that plays a central role in the regulation of growth, maturation, and aging and is subdivided into 'Shen yang' (kidney 'yang') and 'Shen yin' (kidney 'yin'). Kidney 'yang' can be described as the driving force of all metabolic processes that improve the movements of the body. The production of kidney 'yin' is considered to be effective in increasing nutrition to the muscles and improving the smoothness of body movements by constituting the constructive potential for the production of kidney 'yang'. Based on this concept, TCM aims to potentiate the diminishing vitality of this transformative cycle caused by a decline in the essence ('Jing'), which is stored in the kidneys and underpins the functions of both kidney 'yin' and 'yang' [7]. In TCM theory, most PD patients (about two thirds) belong to 'deficiency in "yin" or "Jing" and "yang" or "Qi" in "Gan" (liver) and "Shen" (kidney)' by TCM 'Zheng' classification, whereas the other 'Zheng' such as blood stasis, deficiency in 'Qi' with blood, and phlegm are much less than the first 'Zheng' [15].

\section{TCM Treatment in PD}

Our consensus guideline suggests that decoction with medicinal ingredients or Chinese patent medicine tonifying the 'yin' and 'yang' of 'Gan' (liver) and 'Shen' (kidney) may be useful to treat or improve rigidity, tremor, bradykinesia, sleep disorders, constipation, depression, anorexia, and automatic symptoms using 'Di-Huang-Yin-Z' (地黃飲子), 'Da-Ding-Feng-Zhu' (大定風珠), 'Zhen-Gan-Xi-Feng-Tang' (鎮肝熄風湯), 'Tian-Ma-Gou-Teng-Yin' (天麻勾藤飲), 'Da-Bu-Yin-Wan' (大補陰丸), 'Ren-Shen-Yang-Rong-Tang' (人參養榮湯), and others, according to the 'Zheng' classification of PD [15]. Other TCM decoctions or patent medicines such as 'DaoTan-Tang' (導痰湯), 'Tian-Wang-Bu-Xin-Dan' (天王補心丹), 'Gui-Pi-Tang' (歸脾湯), ‘Bu-YangHuan-Wu-Tang' (補陽還五湯), 'Qi-Ju-Di-Huang-Tang' (杞菊地黃丸), 'Ma-Ren-Wan' (麻仁丸), 'Yang-Xue-Qing-Nao-Ke-Li' (養血清腦顆粒), and others may improve some of the other nonmotor symptoms of the other 'Zheng' classification patients with PD. If the TCM doctor or neurologist is an expert in TCM, he may make an individual prescription choosing ingredients according to the individual manifestation of 'Zheng' and the 'pulse' properties of the patient. All of the TCM therapies can be used for all stages and periods of PD [7, 15-18]; if the patient suffers from dysphagia, TCM medication can be taken via a nasogastric tube.

Acupuncture and 'Tui na' (推拿, TCM massage) are often used as complementary treatment for PD patients with motor and non-motor symptoms; sometimes, the two therapies are used in early-onset PD as initial management to delay disease progression [19]. Acupuncturists and massagers may choose acupoints according to TCM theory which may tonify the 'yin' and 'yang' of 'Gan' and 'Shen' or which may prompt the blood circulation of the body and/or remove blood stasis. The acupoints normally used are 'Chen Shan' (承山, BL57), 'Tai Xi' (太溪, KI3), 'Tai Chong' (太衝, LR3), 'Shen Shu' (腎腧, BL23), 'Zu San Li' (足三里, ST36), 'San Yin Jiao' (三陰交, SP6), 'Xue Hai' (血海, SP10), 'Yang Lin Quan' (陽陵泉, GB34), 'He Gu' (合谷, LI4), 'Qu Chi' (曲池, LI11), 'Du Bi' (犢鼻, ST35), 'Shou San Li' (手三里, LI10), 'Pi Shu' 
(脾腧, BL20), 'Gan Shu' (肝腧, BL18), 'Wei Zhong' (委中, BL40), 'Yin Lin Quan' (陰陵泉, SP9), 'Yao Yang Guan' (腰陽關, DU3), 'Ming Men' (命門, DU4), 'Qi Hai' (氣海, RN6), and 'Guan Yuan' (關元, RN4) [20-26].

Tai chi chuan is an effective treatment improving the stability of PD patients [27-29] and can be used to control some of the non-motor symptoms such as automatic impairment, sleep disorders, and depression. Any type of Tai chi chuan can be used for the improvement of the disease. Those PD patients who cannot keep a standing posture for the exercise of Tai chi chuan may remain seated until they are gradually able to stand up for the training [28-30]. The core of Tai chi chuan is to move the body by mind direction slowly, which differs substantially from aerobic and strength exercises [28].

\section{Other Integrative Treatment or Exercises}

\section{Surgery}

Most patients in the mild stage or with early-onset PD react well to anti-parkinsonism treatment and/or TCM and/or CAM. Surgery may be used in two situations. Firstly, with the progression of $\mathrm{PD}$, motor fluctuation, dyskinesia, and even on-off phenomenon will occur, and DBS will be used to improve the movement disorders of the patients [11]. Secondly, if the patients suffer from very heavy motor symptoms such as tremor or rigidity, or the medication used shows a lack of efficacy, they may start treatment with DBS. We emphasize that DBS is most effective against tremor and rigidity and is not the first choice for posture and balance disorders. Age and the course of the disease are the selective factors for treatment with DBS $[11,12]$.

\section{Aerobic Exercise, Strength Exercise, Yoga, Hyperbaric Oxygen, Rehabilitation,}

Psychological Guidance, and Health Care

These integrative therapies or exercises are helpful in improving rigidity, balance disorder, and parts of the non-motor symptoms, and some patients have self-initiated these activities or treatments during all periods or stages of anti-parkinsonism treatment [31-33]. Psychological guidance is useful to reduce depression, and health care is important to prevent inflammation, nutrition impairment, falls, and fractures. These exercises or therapies are not the main treatment for improving the symptoms of PD [34-40].

\section{Supplementary Treatment}

Ginkgo supplementary, Ginseng supplementary, Rhodiola rosea supplementary, melatonin, deep sea fish oil, and American ginseng are often used to improve fatigue, memory impairment, weakness, automatic impairment, and sleep disorders of PD patients, suggested by family members or patients themselves [41, 42]. These CAM are not harmful, but there is still no evidence-based research supporting their intake except for melatonin [43-45].

\section{Preventive Treatment}

PD is a progressive neurodegenerative disease; previous to morbidity, most patients have various preclinical symptoms such as sleep disorders, restless leg syndrome, olfactory dysfunction, and cognitive impairment. With the progression of these symptoms, patients tend to suffer from PD; our consensus guideline suggests that these patients should be treated in the preclinical stage to slow down or stop disease progression. Preventive treatment has two implications $[44,45]$ : firstly, to reduce or stop suffering from the disease, and secondly, to slow down the progression of PD. The TCM compound cream formula (膏方), herbs which 
may tonify the 'yin' and 'yang' functions of 'Gan' and 'Shen', vitamin E, Ginkgo supplementary, Ginseng supplementary, $R$. rosea supplementary, melatonin, deep sea fish oil, and American ginseng may partly present alternative preventive therapies $[2,46,47]$, but there is still a lack of evidence-based research.

\section{Conclusion}

There are no absolute fixed models for the management or treatment of PD, and thus, patients should be treated individually according to their condition, which may have its own individual sensitivity and specificity for each patient. Even the same patient may be treated by different medication in different stages of the disease. The choice of diagnostic methods and therapeutic strategies for PD varies among physicians, general neurologists, and movement disorder specialists. Furtherconsensus programs on the diagnosis and management of PD patients are warranted, especially for physicians and general neurologists caring for PD patients in China. The aim and scope of this consensus guideline was to emphasize that neurologists and physicians (including TCM doctors) should pay attention to integrative therapy in the treatment of PD patients.

\section{References}

$>1$ Olanow CW, Rascol O, Hauser R, Feigin PD, et al: A double-blind, delayed-start trial of rasagiline in Parkinson's disease. N Engl J Med 2009;361:1268-1278.

$\checkmark 2$ Chen X, Pan W: The treatment strategies for neurodegenerative diseases by integrative medicine. Integr Med Int 2014;1:223-225.

-3 Hely MA, Morris JG, Reid WG, Trafficante R: Sydney Multicenter Study of Parkinson's disease: non-L-doparesponsive problems dominate at 15 years. Mov Disord 2005;20:190-199.

4 Lu H, Pan W, Wang J, Wu C, et al: The current status of integrative therapies in treating Parkinson's disease in six general hospitals in Shanghai. Int J Integr Med 2013;1:17.

5 Chen W, Chen S, Xiao Q, Wang G, Chen SD: Current clinical practice for Parkinson's disease among Chinese physicians, general neurologists and movement disorders specialists: a national survey. BMC Neurol 2012;12:155.

6 Chinese Parkinson's Disease and Movement Disorders Society of Chinese Medical Association: The guideline of the treatment for Parkinson's disease in China (third edition). Chin J Neurol 2014;47:428-433.

7 Pan W, Kwak S, Liu Y, Sun Y, Fang Z, Qin B, Yamamoto Y: Traditional Chinese medicine improves activities of daily living in Parkinson's disease. Parkinsons Dis 2011;2011:789506.

8 Pan W, Kwak S, Li G, Chen Y, Cai D: Therapeutic effect of Yang-Xue-Qing-Nao granules on sleep dysfunction in Parkinson's disease. Chin Med 2013;8:14.

-9 Pan W, Liu J, Wang Q, et al: Clinical Study on chronic pain in Parkinson's disease. Integr Med Int 2014;1:93-101.

10 Pan W, Kwak S, Liu Y, Fang Z, Zhu X, Yamamoto Y: A compound belonging to traditional Chinese medicine improves nocturnal activity in Parkinson's disease. Sleep Med 2011;12:307-308.

11 Ye W, Norris JM: Deep brain stimulation for Parkinson disease in elderly individuals. JAMA Neurol 2015;72: 367-368.

12 Odekerken VJ, Boel JA, Geurtsen GJ, Schmand BA, Dekker IP, de Haan RJ, Schuurman PR, de Bie RM; NSTAPS Study Group: Neuropsychological outcome after deep brain stimulation for Parkinson disease. Neurology 2015;84:1355-1361.

13 Athauda D, Foltynie T: The ongoing pursuit of neuroprotective therapies in Parkinson disease. Nat Rev Neurol 2015;11:25-40.

14 Fahn S: The medical treatment of Parkinson disease from James Parkinson to George Cotzias. Mov Disord 2015;30:4-18.

15 Gu C, Yuan C: The study on distribution of Zheng classification for Parkinson's disease and the treatment regular pattern in traditional Chinese medicine. Shang Hai Zhong Yi Yao Za Zhi 2013;7:12-14.

-16 Zhang G, Xiong N, Zhang Z, Liu L, Huang J, Yang J, Wu J, Lin Z, Wang T: Effectiveness of traditional Chinese medicine as an adjunct therapy for Parkinson's disease: a systematic review and meta-analysis. PLoS One 2015;10:e0118498.

17 Li M, Yang MH, Liu Y: Effects of Chinese herbal medicine Bushenhuoxue Granule on quality of life of patients with Parkinson disease: a randomized, double-blinded and placebo-controlled trial (in Chinese). Zhong Xi Yi Jie He Xue Bao 2012;10:310-317. 
Pan et al.: A Practical Consensus Guideline for the Integrative Treatment of Parkinson's Disease in Shanghai, China

18 Parkinson's disease outpatients: a pilot study on efficacy, tolerability, and quality of life. J Altern Complement Med 2006;12:395-399.

20 Wang H, Liang X, Wang X, Luo D, Jia J, Wang X: Electro-acupuncture stimulation improves spontaneous locomotor hyperactivity in MPTP intoxicated mice. PLoS One 2013;8:e64403.

21 He X, Yan T, Chen R, Ran D: Acute effects of electro-acupuncture (EA) on hippocampal long term potentiation (LTP) of perforant path-dentate gyrus granule cells synapse related to memory. Acupunct Electrother Res 2012;37:89-101.

22 Lu ZY, Zhao H, Wang T, Chen J, Zong L: Effects of acupuncture on behavior and striatal apoptosis in mice with Parkinson disease (in Chinese). Zhen Ci Yan Jiu 2012;37:186-190.

-23 Chen YL, Feng WJ, Zhang XL: Parkinson's disease combined with overactive bladder syndrome treated with acupuncture and medication (in Chinese). Zhongguo Zhen Jiu 2012;32:215-218.

24 Chang XH, Zhang LZ, Li YJ: Observation on therapeutic effect of acupuncture combined with medicine on Parkinson disease (in Chinese). Zhongguo Zhen Jiu 2008;28:645-647.

-25 Ulm G: Differential therapy of advanced Parkinson's disease with special reference to complementary therapeutic approaches. Praxis (Bern 1994) 2004;93:1869-1872.

-26 Huang Y, Jiang XM, Li DJ: Effects on electro-scalp acupuncture on cerebral dopamine transporter in patients with Parkinson's disease (in Chinese). Zhongguo Zhong Xi Yi Jie He Za Zhi 2006;26:303-307.

-27 Hackney ME, Earhart GM: Tai Chi improves balance and mobility in people with Parkinson disease. Gait Posture 2008;28:456-460.

28 Li F, Harmer P, Liu Y, Eckstrom E, Fitzgerald K, Stock R, Chou LS: A randomized controlled trial of patientreported outcomes with tai chi exercise in Parkinson's disease. Mov Disord 2014;29:539-545.

-29 Li F, Harmer P, Fitzgerald K, Eckstrom E, Stock R, Galver J, Maddalozzo G, Batya SS: Tai chi and postural stability in patients with Parkinson's disease. N Engl J Med 2012;366:511-519.

$\$ 30$ Li F: Tai Ji Quan exercise for people with Parkinson's disease and other neurodegenerative movement disorders. Int J Integr Med 2013, DOI: 10.5772/56243.

-31 Uc EY, Doerschug KC, Magnotta V, Dawson JD, Thomsen TR, Kline JN, Rizzo M, Newman SR, Mehta S, Grabowski TJ, Bruss J, Blanchette DR, Anderson SW, Voss MW, Kramer AF, Darling WG: Phase I/II randomized trial of aerobic exercise in Parkinson disease in a community setting. Neurology 2014;83:413-425.

-32 Tabak R, Aquije G, Fisher BE: Aerobic exercise to improve executive function in Parkinson disease: a case series. J Neurol Phys Ther 2013;37:58-64.

-33 Abrantes AM, Friedman JH, Brown RA, Strong DR, Desaulniers J, Ing E, Saritelli J, Riebe D: Physical activity and neuropsychiatric symptoms of Parkinson disease. J Geriatr Psychiatry Neurol 2012;25:138-145.

-34 Schenkman M, Hall DA, Barón AE, Schwartz RS, Mettler P, Kohrt WM: Exercise for people in early- or mid-stage Parkinson disease: a 16-month randomized controlled trial. Phys Ther 2012;92:1395-1410.

-35 Haralur SB: Clinical strategies for complete denture rehabilitation in a patient with Parkinson disease and reduced neuromuscular control. Case Rep Dent 2015;2015:352878.

-36 Ory Magne F, Fabre N, Gu C, Pastorelli C, Tardez S, Marchat JC, Marque P, Brefel Courbon C: An individual rehabilitation program: evaluation by Parkinsonian patients and their physiotherapists. Rev Neurol (Paris) 2014; 170:680-684.

-37 Sihvonen AJ, Leo V, Särkämö T, Soinila S: Effectiveness of music in brain rehabilitation. A systematic review (in Finnish). Duodecim 2014;130:1852-1860.

-38 Pierobon A, Giardini A, Maestri R, Farina C, Callegari S, Torlaschi V, Bertotti G, Majani G, Frazzitta G: Disexecutive functions and depression in patients with Parkinson disease: the impact on rehabilitation outcome. Am J Phys Med Rehabil 2014;93:764-773.

-39 Abrantes AM, Friedman JH, Brown RA, Strong DR, Desaulniers J, Ing E, Saritelli J, Riebe D: Physical activity and neuropsychiatric symptoms of Parkinson disease. J Geriatr Psychiatry Neurol 2012;25:138-145.

-40 Wahbeh H, Elsas SM, Oken BS: Mind-body interventions: applications in neurology. Neurology 2008;70:23212328.

-41 Cao F, Sun S, Tong ET: Experimental study on inhibition of neuronal toxical effect of levodopa by Ginkgo biloba extract on Parkinson disease in rats. J Huazhong Univ Sci Technolog Med Sci 2003;23:151-153.

-42 Yang SF, Wu Q, Sun AS, Huang XN, Shi JS: Protective effect and mechanism of Ginkgo biloba leaf extracts for Parkinson disease induced by 1-methyl-4-phenyl-1,2,3,6-tetrahydropyridine. Acta Pharmacol Sin 2001;22: 1089-1093.

43 Miller E, Morel A, Saso L, Saluk J: Melatonin redox activity. Its potential clinical applications in neurodegenerative disorders. Curr Top Med Chem 2015;15:163-169.

-44 Breen DP, Vuono R, Nawarathna U, Fisher K, Shneerson JM, Reddy AB, Barker RA: Sleep and circadian rhythm regulation in early Parkinson disease. JAMA Neurol 2014;71:589-595.

$\checkmark 45$ Videnovic A, Noble C, Reid KJ, Peng J, Turek FW, Marconi A, Rademaker AW, Simuni T, Zadikoff C, Zee PC: Circadian melatonin rhythm and excessive daytime sleepiness in Parkinson disease. JAMA Neurol 2014;71: 463-469.

-46 Pan W: Integrative reserve and integrative medicine. Integr Med Int 2014;1:127-129.

47 Muzino Y: Recent research progress in and future perspective on treatment of Parkinson's disease. Integr Med Int 2014;1:67-79. 